\title{
Characteristics of hip involvement in patients with ankylosing spondylitis in Korea
}

Hyemin Jeong, Yeong Hee Eun, In Young Kim, Hyungjin Kim, Jaejoon Lee, Eun-Mi Koh, and Hoon-Suk Cha

Department of Medicine, Samsung Medical Center, Sungkyunkwan University School of Medicine, Seoul, Korea

Received: July 21, 2015

Revised : August 25, 2015

Accepted: September 12, 2015

\section{Correspondence to}

Hoon-Suk Cha, M.D.

Division of Rheumatology,

Department of Medicine,

Samsung Medical Center,

Sungkyunkwan University

School of Medicine, 81 Irwon-ro,

Gangnam-gu, Seoul 06351, Korea

Tel: $+82-2-3410-3435$

Fax: +82-2-3410-3849

E-mail: hoonsuk.cha@samsung.com
Background/Aims: To evaluate the clinical course of hip arthritis and the risk factors for hip joint replacement in patients with ankylosing spondylitis (AS).

Methods: In this retrospective analysis, we evaluated $488 \mathrm{AS}$ patients at a single tertiary hospital. At baseline and the most recent visit to the outpatient clinic from the patients with hip arthritis in AS, radiographic hip arthritis was evaluated using the Bath Ankylosing Spondylitis Radiology Hip Index (BASRI-h). Also the average of the hip joint space width (interbone distance) at three distinct sites between the acetabulum and femoral head was recorded.

Results: Among 488 patients with AS, 60 patients (12.3\%) had hip arthritis. Erythrocyte sedimentation rate were associated with hip involvement (odds ratio, 1.o1; 95\% confidence interval [CI], 1.00 to 1.02; $p=0.004)$. Long disease duration and advanced axial disease were associated with severe hip arthritis ( $3 \geq$ BASRI-h) at baseline. BASRI-h and interbone distance did not significantly change in patients with hip involvement during the follow-up period of $81.4 \pm 35.7$ months. Five patients had hip joint replacement surgery during follow-up period. The body mass index (BMI) and BASRI-h at baseline were associated with joint replacement surgery (hazard ratio [HR], 1.30; $95 \% \mathrm{CI}, 1.00$ to 1.67; $p=0.049$ and $\mathrm{HR}$, 20.64; $95 \%$ CI, 2.39 to $178.11 ; p=0.006$, respectively).

Conclusions: Most of the patients with hip arthritis in AS showed no significant radiographic progression during the follow-up period. High BMI and advanced hip arthritis at baseline were associated with hip joint replacement surgery in patients with AS.

Keywords: Spondylitis, ankylosing; Hip; Body mass index

\section{INTRODUCTION}

Ankylosing spondylitis (AS) is a chronic inflammatory disease that primarily affects the axial skeleton. Hip involvement is common in AS patients and the reported prevalence of clinical hip involvement in AS is from $24 \%$ to $36 \%$ and prevalence of radiographic hip arthritis ranges from $9 \%$ to $22 \%$ [1-3]. Unlike new bone formation in axial spine, synovial inflammation within the hip joint causes bone erosion and joint space narrowing. The histological appearance of the synovial membrane in AS is characterized by synovial lining cell hyperplasia and lymphoid cell aggregates, similar to those observed in rheumatoid arthritis (RA) [4]. Progressive flexion deformity and destruction of hip joint might also be observed. Eventually, hip involvement causes functional impairment and disability in patients with AS [5]. Previous studies have reported that early onset of disease 
and axial disease are associated with hip joint involvement and hip replacement surgery in $\mathrm{AS}[1,3]$. It has been reported that $5 \%$ of AS patients need hip replacement surgery [1]. In a recent study, the total joint replacement surgery rate in patients with RA decreased $40 \%$ with the widespread use of anti-tumor necrosis factor (anti-TNF) agents, whereas the total joint replacement surgery rate in spondyloarthritis increased 40\% [6]. Despite the worse functional outcome in patients with hip involvement, there are few studies concerning hip arthritis compared with the axial disease in AS. The aim of the present study was to investigate the clinical course of hip arthritis and the risk factors for hip joint replacement in AS.

\section{METHODS}

\section{Study population}

The subjects were selected from a pool of patients who first visited the rheumatology outpatient clinic of Samsung Medical Center in Seoul, South Korea between January 2001 and January 2011 in a consecutive manner. We selected patients diagnosed with AS based on fulfillment of the modified New York criteria [7]. We excluded the patients who underwent total hip replacement surgery for both hips before the initial visit at the outpatient clinic. Patients who had unilateral hip replacement surgery were included. Clinical characteristics and laboratory data were collected retrospectively. Peripheral arthritis included the knee, ankle, foot, elbow, wrist and hand joints. Disease duration was defined as the time from the symptom onset to the first visit to the outpatient clinic. The follow-up duration was defined as time from the initial visit to the last visit at the clinic. $\mathrm{X}$-ray follow-up duration was the time from the baseline $\mathrm{X}$-ray to the most recent image taken. Hip involvement at baseline was defined according to the clinical opinion of the rheumatologist, such as pain and limited range of motion and radiological evidence of hip arthritis, defined by at least the score of 1 in the Bath Ankylosing Spondylitis Radiology Hip Index (BASRI-h) scoring system [8]. This study was approved through the Institutional Review Board of Samsung Medical Center.

\section{Radiographs and scoring}

Paired recent radiographs and baseline pelvis X-ray were analyzed in 60 patients with hip arthritis. Two trained rheumatologists (HJ and IYK) scored the radiographic hip arthritis using the BASRI-h scoring system (grade o to 4). We also used published methods to measure the average interbone distance between the acetabulum and femoral head of both hips at three distinct sites: (1) at 2 $\mathrm{mm}$ inner of the external end of the acetabulum; (2) at the vertical line through the femoral head center; and (3) at the head-neck center line [9]. Among 60 patients with hip arthritis, five patients had unilateral hip involvement. The BASRI-h grade and interbone distance were calculated by the mean of the right and left hips except for the five patients who had unilateral hip replacement surgery before the initial visit. In these five patients who underwent unilateral hip replacement surgery before the initial visit, the artificial joint was not included and only one side of the joint was used for analysis. In addition, when patients underwent hip replacement surgery during the follow-up period, BASRI-h grade became 4 and interbone distance became $\mathrm{O}$ and mean value of the both hip joints were used for the analysis.

Severe radiographic hip involvement was defined as $3 \geq$ BASRI-h grades at baseline. A change in the BASRI-h and interbone distance between the baseline and the most recent image was divided by time intervals between the time of baseline and the most recent image was taken. The changes were calculated after subtracting the value of the baseline image from the value of the recent images. Therefore, in case of radiographic progression, $\triangle$ BASRI-h value becomes positive and $\Delta$ mean of interbone distance value becomes negative. The sacroiliitis grade was defined as the sum of both the right and left sacroiliitis grades according to the modified New York criteria [7]. The presence of syndesmophytes at baseline was also assessed. The mean value of two readers was used for analysis. The intra-class correlation coefficient of the BASRI-h grade of two readers was 0.927 (95\% confidence interval [CI], 0.91 to $0.94 ; p<0.001$ ), and the interbone distance value was 0.973 (95\% CI, 0.97 to $0.98 ; p<0.001)$.

\section{Statistical analysis}

Descriptive statistics were used to identify the characteristics of the study population. Categorical variables were compared between groups using the chi-square test or Fisher exact test. For continuous variables, the data were 
presented as the mean $\pm \mathrm{SD}$, and variables were coma pared using $t$ tests. Univariable logistic regression analyses were performed to identify the predictors associated with hip involvement. Cox proportional hazards models were used to estimate the hazards ratio (HRs) with $95 \%$ CI of hip replacement surgery in patients with hip involvement. Schoenfeld residuals were examined for the covariates, and significant departures from the proportional hazards assumption were not detected. Variables associated with $p$ value outcomes $\leq 0.10$ were included in the multivariable analysis. Because the follow-up duration was different between patients, generalized estimating equation analysis was performed to assess the changes in the BASRI-h, interbone distance, erythrocyte sedimentation rate (ESR), C-reactive protein (CRP) level, and body mass index (BMI) over the follow-up period. We used an exchangeable correlation structure as a working correlation in repeated-measurement design. SAS version 9.4 (SAS Institute, Cary NC, USA) and SPSS version 19.0 (IBM Co., Armonk, NY, USA) were used for analysis. Statistical significance was considered for $p<$ 0.05 .

\section{RESULTS}

In total, 488 patients with AS were included in the analysis. The baseline characteristics of patients are shown in Table 1. Among the 488 patients, 60 patients (12.3\%) had hip involvement. In univariable analysis, ESR was associated with hip involvement in AS (odds ratio [OR], 1.01; 95\% CI, 1.00 to 1.02; $p=0.004$ ) (Table 2). The age at disease onset, sex, disease duration, and smoking status were not significantly associated with hip involvement. All the patients used nonsteroidal anti-inflammatory drugs at the initial treatment. Follow-up duration after the initial visit was longer in patients with hip arthritis than patients without $(97.4 \pm 38.4$ months vs. $67.5 \pm$ 55.3 months, $p<0.001$ ). During the follow-up period, patients with hip arthritis used more anti-TNF agents than patients without hip arthritis (48.3\% vs. $23.4 \%, p<$ o.001).

Among the 60 AS patients with hip arthritis, the mean BASRI-h grade was $2.0 \pm 0.7$, and the mean interbone distance was $2.9 \pm 1.0 \mathrm{~mm}$ at baseline. Of the 60 patients, 10 patients $(16.7 \%)$ had BASRI-h grade 3 or 4 and were
Table 1. Baseline characteristics of patients $(n=488)$

\begin{tabular}{lc}
\hline Variable & Value \\
\hline Age at disease onset, yr & $25.9 \pm 9.2$ \\
Male sex & $408(83.6)$ \\
Hip involvement & $60(12.3)$ \\
Disease duration, mon & $39(12-114)$ \\
Peripheral arthritis, ever & $121(24.8)$ \\
Enthesitis, ever & $49(10.0)$ \\
Uveitis, ever & $102(20.9)$ \\
Syndesmophytes & $127(26)$ \\
Sacroiliitis grade ${ }^{\mathrm{a}}$ & $4.9 \pm 1.7$ \\
Body mass index, $\mathrm{kg} / \mathrm{m}^{2}$ & $23.3 \pm 3.3$ \\
Erythrocyte sediment rate, $\mathrm{mm} / \mathrm{hr}$ & $37.8 \pm 28.7$ \\
C-reactive protein, $\mathrm{mg} / \mathrm{dL}$ & $1.01(0.33-2.56)$ \\
HLA-B27 positive $(\mathrm{n}=365)$ & $337(92.3)$ \\
Current smoker & $49(10.0)$ \\
\hline
\end{tabular}

Values are presented as mean $\pm \mathrm{SD}$, number (\%), or median (interquartile range).

HLA, human leukocyte antigen.

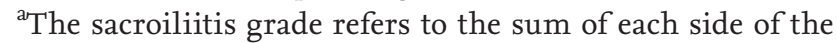
sacroiliitis grade according to the modified New York criteria.

classified as with severe hip arthritis and other 50 patients (83.3\%) with BASRI-h grade 1 or 2 were classified as not having severe hip arthritis. Patients with severe hip arthritis had longer disease duration (142.8 \pm 93.8 months vs. $55.2 \pm 64.9$ months, $p=0.001$ ) and had more advanced axial disease (sum of each side sacroiliitis grade was $6.8 \pm 1.7$ vs. $5.5 \pm 1.4, p=0.014$; and presence of syndesmophyte was $70 \%$ vs. $20 \%, p=0.003$ ) than patients with no severe hip arthritis.

In the 60 patients with hip arthritis, the mean of X-ray follow-up duration was $81.4 \pm 35.7$ months, with a median value of 78 months (range, 23 to 198). We observed no significant radiographic changes for the hip joint during the follow-up period. The value of both the BASRI-h $(p=$ $0.138)$ and interbone distance $(p=0.296)$ were not significantly changed from the baseline to the time of the most recent image taken. Among the 60 patients with hip involvement, five patients (8.3\%) underwent joint replacement surgery during the follow-up period. Patients with hip joint replacement surgery showed advanced hip arthritis and higher BMI at baseline compared with patients without hip replacement surgery $\left(27.8 \pm 3.1 \mathrm{~kg} / \mathrm{m}^{2}\right.$ vs. $\left.23.4 \pm 4.2 \mathrm{~kg} / \mathrm{m}^{2}, p=0.029\right)$. And initial BASRI-h grade 

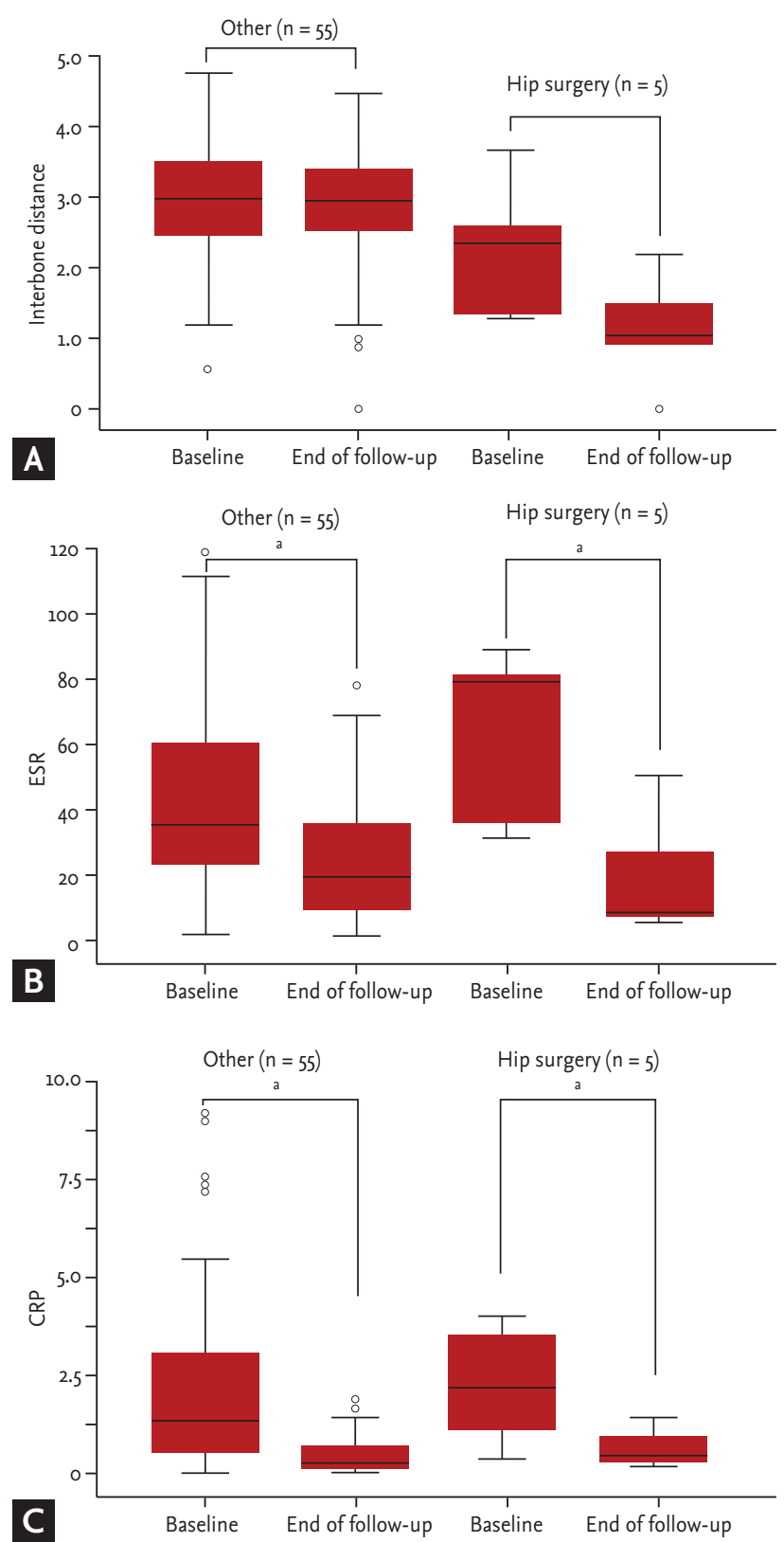

Figure 1. Changes in the interbone distance and acute phase reactant levels in patients with hip arthritis. (A) The interbone distance at baseline and at the end of the follow-up period in patients with and without hip replacement surgery. Changes in (B) erythrocyte sediment rate (ESR) and (C) $\mathrm{C}$-reactive protein (CRP) at baseline and the end of the follow-up period in patients with and without hip replacement surgery. ${ }^{\mathrm{a}} \mathrm{p}<0.01$.

was higher in patients with hip joint replacement surgery $(2.6 \pm 0.4$ vs. $1.8 \pm 0.7, p=0.012)$. During the follow-up period, hip joint progression was faster in patients with hip replacement surgery than patients without surgery
( $\triangle$ BASRI-h/year: $0.16 \pm 0.09$ vs. $0.02 \pm 0.06, p<0.001$; and $\Delta$ interbone distance/year: $-0.19 \pm 0.04$ vs. $-0.02 \pm 0.07, p<$ 0.001). Table 3 shows factors associated with hip replacement surgery among patients with hip arthritis in AS. Multivariable analysis revealed that both BMI and initial BASRI-h were associated with hip joint replacement surgery. In addition, among the 422 patients without hip arthritis at baseline, no patients showed significant radiographic changes or had hip replacement surgery during the follow-up period.

Changes in the interbone distance and acute phase reactant in patients with hip arthritis are shown in Fig. 1. The interbone distance for five patients with hip surgery decreased $(p=0.091)$ and was not significantly affected in 55 other patients. ESR and CRP were significantly decreased at the end of follow-up from the baseline, regardless of hip replacement surgery. In addition, ESR and CRP were significantly decreased in all 488 patients during the follow-up period, regardless of hip joint involvement. BMI was not changed significantly during the follow-up period regardless of hip joint involvement or hip replacement surgery.

\section{DISCUSSION}

In the present study, we attempted to identify the radiographic changes in the hip joint and evaluate the associated factors of hip arthritis in AS. Our study showed that the prevalence of hip involvement was $12.3 \%$ and severe radiographic hip arthritis were associated with longer disease duration and advanced axial disease, which corresponds well with previous studies [3,10]. We found that higher BMI was associated with hip replacement surgery. These results are in agreement with the RESISPONSER cohort, which showed that BMI is higher in patients with hip replacement surgery in AS compared with those without surgery [1]. Ottaviani et al. [11] reported that high BMI negatively influences the response to infliximab in AS. Patients with psoriatic arthritis (PsA) have higher mean BMI than the general population [12]. Also obese patients with PsA are less likely to achieve sustained minimal disease activity compared with patients of normal weight [13]. An association between obesity and inflammation has been suggested. The levels of adipokines, such as leptin, TNF- $\alpha$ and interleukin 6 
Table 2. Factors associated with hip involvement in patients with ankylosing spondylitis

\begin{tabular}{llc}
\hline Variable & OR $(95 \%$ CI $)$ & $p$ value \\
\hline Age at disease onset, yr & $0.981(0.950-1.013)$ & 0.235 \\
Male sex & $0.978(0.473-2.020)$ & 0.951 \\
Disease duration, mon & $0.999(0.996-1.003)$ & 0.627 \\
Peripheral arthritis & $0.732(0.375-1.428)$ & 0.360 \\
Enthesitis, ever & $0.793(0.302-2.087)$ & 0.639 \\
Uveitis, ever & $0.831(0.415-1.664)$ & 0.602 \\
Syndesmophytes & $1.254(0.693-2.270)$ & 0.454 \\
Sacroiliitis grade ${ }^{\mathrm{a}}$ & $1.099(0.943-1.282)$ & 0.225 \\
Body mass index, $\mathrm{kg} / \mathrm{m}^{2}$ & $1.057(0.975-1.145)$ & 0.181 \\
Erythrocyte sediment rate, $\mathrm{mm} / \mathrm{hr}$ & $1.012(1.004-1.021)$ & 0.004 \\
C-reactive protein, $\mathrm{mg} / \mathrm{dL}$ & $1.082(0.987-1.187)$ & 0.093 \\
HLA-B27 positive $(\mathrm{n}=365)$ & $3.229(0.426-24.481)$ & 0.257 \\
Current smoker & $1.214(0.519-2.840)$ & 0.655 \\
\hline
\end{tabular}

OR, odds ratio; CI, confidence interval; HLA, human leukocyte antigen.

${ }^{a}$ The sacroiliitis grade refers to the sum of each side of the sacroiliitis grade according to the modified New York criteria.

Table 3. Factors associated with hip replacement surgery in ankylosing spondylitis patients with hip involvement

\begin{tabular}{|c|c|c|c|c|}
\hline \multirow{2}{*}{ Clinical characteristic } & \multicolumn{2}{|c|}{ Univariable model } & \multicolumn{2}{|c|}{ Multivariable model } \\
\hline & $\mathrm{HR}(95 \% \mathrm{CI})$ & $p$ value & $\operatorname{HR}(95 \% \mathrm{CI})$ & $p$ value \\
\hline Age at disease onset & $1.003(0.976-1.031)$ & 0.823 & & \\
\hline Male sex & $0.651(0.072-5.882)$ & 0.702 & & \\
\hline Peripheral arthritis & $1.615(0.246-10.603)$ & 0.618 & & \\
\hline Syndesmophyte & $1.503(0.244-9.253)$ & 0.660 & & \\
\hline Sacroiliitis grade ${ }^{a}$ & $1.088(0.597-1.984)$ & 0.783 & & \\
\hline Body mass index & $1.157(0.991-1.352)$ & 0.066 & $1.295(1.001-1.674)$ & 0.049 \\
\hline Erythrocyte sediment rate & $1.019(0.992-1.047)$ & 0.175 & & \\
\hline C-reactive protein & $0.983(0.689-1.402)$ & 0.924 & & \\
\hline BASRI-h (initial) & $13.142(2.050-84.241)$ & 0.007 & $20.643(2.392-178.110)$ & 0.006 \\
\hline Interbone distance (initial), mm & $0.497(0.202-1.222)$ & 0.128 & & \\
\hline Anti-TNF agent use & $1.000(0.005-218.762)$ & 1.000 & & \\
\hline
\end{tabular}

HR, hazard ratio; CI, confidence interval; BASRI-h, Bath Ankylosing Spondylitis Radiology Hip Index; TNF, tumor necrosis factor.

${ }^{a}$ The sacroiliitis grade refers to the sum of each side of the sacroiliitis grade according to the modified New York criteria.

positively correlate with BMI, and the concentrations of adipokines are increased in patients with metabolic syndrome [14]. A recent study revealed that elevated serum adipokines are associated with radiographic damage in AS [15]. The systemic overload of adipokines has been associated with chronic inflammation, and this condition could be applicable to the involvement of hip arthritis in AS. Biomechanical factors could influence radiographic progression. Mechanical strain drives entheseal inflammation and new bone formation in spondyloarthritis via the Erk 1/2 signaling pathway [16]. It has been reported that obesity is increased in patients with primary total hip arthroplasty [17]. Thus, the high incidence of hip arthritis progression resulting in hip joint replacement surgery in obese patients might reflect inflammation associated with mechanotransduction and immune re- 
sponse factors.

In the present study, most of the patients showed no significant radiographic changes during the follow-up period. Among the 60 AS patients with hip arthritis, only five patients showed radiographic progression and underwent joint replacement surgery and the other 55 patients showed almost no radiographic changes during the follow-up period. Advanced hip joint damage and high BMI at baseline were risk factors for the hip joint replacement surgery. Patients in our study had visited the rheumatology outpatient clinic regularly and received standard treatment for AS such as non-steroidal anti-inflammatory drugs or anti-TNF agents. Mean ESR and CRP decreased significantly over the follow-up period, regardless of hip involvement or hip replacement surgery. These results suggest that when AS patients are treated properly, the possibility of significant radiographic hip changes is low. Under these conditions, BMI might influence the radiographic progression of the hip joint. In addition, early initiation of treatment before radiographic hip changes occur may be helpful in preventing hip joint replacement surgery.

This study has some limitations. We conducted a retrospective study, and clinical parameters such as Bath Ankylosing Spondylitis Disease Activity Index, Bath Ankylosing Spondylitis Functional Index, range of joint mobility, or level of daily physical activity were not systemically available. The strength of this study is that we provided the evidence that BMI might influence the outcome of hip arthritis in patients with AS.

In summary, the present study revealed that most of the patients showed no significant radiographic changes during the follow-up period. High BMI and advanced radiographic hip arthritis at baseline were associated with hip joint replacement surgery in patients with AS. Further study is warranted to clarify the role of obesity in the pathogenesis and progression of hip arthritis in AS.

\section{KEY MESSAGE}

1. Most of the patients with hip arthritis in ankylosing spondylitis (AS) showed no significant radiographic progression during the follow-up period.

2. High body mass index and advanced hip ar- thritis at baseline were associated with hip joint replacement surgery in patients with AS.

\section{Conflict of interest}

No potential conflict of interest relevant to this article was reported.

\section{Acknowledgments}

This study was financially supported through a grant from the Korea Health Industry Development Institute (HI14C2285).

\section{REFERENCES}

1. Vander Cruyssen B, Munoz-Gomariz E, Font P, et al. Hip involvement in ankylosing spondylitis: epidemiology and risk factors associated with hip replacement surgery. Rheumatology (Oxford) 2010;49:73-81.

2. Burki V, Gossec L, Payet J, et al. Prevalence and characteristics of hip involvement in spondyloarthritis: a single-centre observational study of 275 patients. Clin Exp Rheumatol 2012;30:481-486.

3. Chen HA, Chen CH, Liao HT, et al. Factors associated with radiographic spinal involvement and hip involvement in ankylosing spondylitis. Semin Arthritis Rheum 2011;40:552-558.

4. Revell PA, Mayston V. Histopathology of the synovial membrane of peripheral joints in ankylosing spondylitis. Ann Rheum Dis 1982;41:579-586.

5. Yılmaz O, Tutoglu A, Garip Y, Ozcan E, Bodur H. Health-related quality of life in Turkish patients with ankylosing spondylitis: impact of peripheral involvement on quality of life in terms of disease activity, functional status, severity of pain, and social and emotional functioning. Rheumatol Int 2013;33:1159-1163.

6. Mertelsmann-Voss C, Lyman S, Pan TJ, Goodman SM, Figgie MP, Mandl LA. US trends in rates of arthroplasty for inflammatory arthritis including rheumatoid arthritis, juvenile idiopathic arthritis, and spondyloarthritis. Arthritis Rheumatol 2014;66:1432-1439.

7. van der Linden S, Valkenburg HA, Cats A. Evaluation of diagnostic criteria for ankylosing spondylitis: a proposal for modification of the New York criteria. Arthritis Rheum 1984;27:361-368. 
8. MacKay K, Brophy S, Mack C, Doran M, Calin A. The development and validation of a radiographic grading system for the hip in ankylosing spondylitis: the bath ankylosing spondylitis radiology hip index. J Rheumatol 2000;27:2866-2872.

9. Konsta M, Sfikakis PP, Bournia VK, Karras D, Iliopoulos A. Absence of radiographic progression of hip arthritis during infliximab treatment for ankylosing spondylitis. Clin Rheumatol 2013;32:1229-1232.

10. Zhao J, Zheng W, Zhang C, Li J, Liu D, Xu W. Radiographic hip involvement in ankylosing spondylitis: factors associated with severe hip diseases. J Rheumatol 2015;42:106110.

11. Ottaviani S, Allanore Y, Tubach F, et al. Body mass index influences the response to infliximab in ankylosing spondylitis. Arthritis Res Ther 2012;14:R115.

12. Bhole VM, Choi HK, Burns LC, et al. Differences in body mass index among individuals with PsA, psoriasis, RA and the general population. Rheumatology (Oxford) 2012;51:552-526.
13. Eder L, Thavaneswaran A, Chandran V, Cook RJ, Gladman DD. Obesity is associated with a lower probability of achieving sustained minimal disease activity state among patients with psoriatic arthritis. Ann Rheum Dis 2015;74:813-817.

14. Deng Y, Scherer PE. Adipokines as novel biomarkers and regulators of the metabolic syndrome. Ann N Y Acad Sci 2010;1212:E1-E19.

15. Syrbe U, Callhoff J, Conrad K, et al. Serum adipokine levels in patients with ankylosing spondylitis and their relationship to clinical parameters and radiographic spinal progression. Arthritis Rheumatol 2015;67:678-685.

16. Jacques $P$, Lambrecht $S$, Verheugen E, et al. Proof of concept: enthesitis and new bone formation in spondyloarthritis are driven by mechanical strain and stromal cells. Ann Rheum Dis 2014;73:437-445.

17. Singh JA, Lewallen DG. Increasing obesity and comorbidity in patients undergoing primary total hip arthroplasty in the U.S.: a 13-year study of time trends. BMC Musculoskelet Disord 2014;15:441. 